\title{
A Bio-inspired Hybrid Cross-Layer Routing Protocol for Energy Preservation in WSN- Assisted IoT
}

\author{
Aditya Tandon ${ }^{1 *}$, Pramod Kumar' ${ }^{1}$, Vinay Rishiwal ${ }^{2}$, Mano Yadav ${ }^{3}$, and Preeti Yadav \\ ${ }^{1}$ Department of Computer Science and Engineering, Krishna Engineering College \\ Ghaziabad, Uttar Pradesh, India \\ [e-mail: aditya.tandon@krishnacollege.ac.in, pramodkumar.hod@krishnacollege.ac.in] \\ ${ }^{2}$ Department of CSIT, MJP Rohilkhand University \\ Bareilly, Uttar Pradesh, India \\ [e-mail: vrishiwal@mjpru.ac.in] \\ ${ }^{3}$ Department of Computer Science, Bareilly College \\ Bareilly, Uttar Pradesh, India \\ [email: vinay.rishiwal.in@ieee.org] \\ ${ }^{4}$ Department of CSIT, MJP Rohilkhand University \\ Bareilly, Uttar Pradesh, India \\ [email: preeti.yadav@mjpru.ac.in] \\ *Corresponding author: Aditya Tandon
}

\begin{abstract}
Nowadays, the Internet of Things (IoT) is adopted to enable effective and smooth communication among different networks. In some specific application, the Wireless Sensor Networks (WSN) are used in IoT to gather peculiar data without the interaction of human. The WSNs are self-organizing in nature, so it mostly prefer multi-hop data forwarding. Thus to achieve better communication, a cross-layer routing strategy is preferred. In the cross-layer routing strategy, the routing processed through three layers such as transport, data link, and physical layer. Even though effective communication achieved via a cross-layer routing strategy, energy is another constraint in WSN assisted IoT. Cluster-based communication is one of the most used strategies for effectively preserving energy in WSN routing. This paper proposes a Bio-inspired cross-layer routing (BiHCLR) protocol to achieve effective and energy preserving routing in WSN assisted IoT. Initially, the deployed sensor nodes are arranged in the form of a grid as per the grid-based routing strategy. Then to enable energy preservation in BiHCLR, the fuzzy logic approach is executed to select the Cluster Head $(\mathrm{CH})$ for every cell of the grid. Then a hybrid bio-inspired algorithm is used to select the routing path. The hybrid algorithm combines moth search and Salp Swarm optimization techniques. The performance of the proposed BiHCLR is evaluated based on the Quality of Service (QoS) analysis in terms of Packet loss, error bit rate, transmission delay, lifetime of network, buffer occupancy and throughput. Then these performances are validated based on comparison with conventional routing strategies like Fuzzy-rule-based Energy Efficient Clustering and Immune-Inspired Routing (FEEC-IIR), Neuro-Fuzzy- Emperor Penguin Optimization (NF-
\end{abstract}


EPO), Fuzzy Reinforcement Learning-based Data Gathering (FRLDG) and Hierarchical Energy Efficient Data gathering (HEED). Ultimately the performance of the proposed BiHCLR outperforms all other conventional techniques.

Keywords: Bio-inspired Algorithms, Cluster Head(CH), Cross-layer, Grid Coordinator, Moth Search (MS), Quality of Service (QoS), Salp Swarm Algorithm (SSA)

\section{Introduction}

$\mathbf{I}_{\mathrm{n}}$

nternet of Things (IoTs) is globally suggested to use in various application for interlinking various networks. In recent days IoTs are used in various heterogeneous networks such as medical network, vehicular network, mobile network as well as sensor networks [1]. In particular, the sensor network or WSN can improve the distributed intelligence and communication protocols for smart devices and various other equipment, which is combined to form a novel futuristic internet solution in IoT. The WSNs are emerging as an advanced platform that is useful in lots of applications like healthcare, environmental monitoring, intelligence surveillance, smart cities, military, etc [2]. In these applications, small sensors act as nodes to collect and transmit the information to a base station or sink node [3]. An individual sensor node is a self-operating device which is connected wirelessly and is spatially distributed [4]. So every individual node can simultaneously sense, process and interact with one another [5]. The price of the IoT systems has decreased dramatically, opening up a number of possibilities to boost potential innovation and deployments [6]. Researchers have been exploring the Wireless Sensor Networks technology (WSNs) for over a decade and, along with numerous routing methods, different techniques have been proposed by the researchers with regard to reducing the packet and frame size of medium access control (MAC) and physical layers. Various other unsophisticated processes, energy combination with applied fusion techniques, time, placement and safety mechanisms have been made aware of. They enrich fundamental infrastructures, high-level abstractions that are assisted by operating system designs, and large-scale management systems to handle data generated in an appropriate way [7]. The energy control of the IoT networks are now becoming self-sufficient [8].

Broadcasting is an efficient method of improving the effectiveness of communication to ensure the data reaches all the designated receiver nodes. The WSN has a multi-hop forwarding nature. The information transmitted over the wireless channel shas a chance for losses, so it is difficult to achieve effective communication in multi-hop forwarding [1]. So the cross-layered routing strategy is an essential requirement for the wireless network, to solve the problems that arise during data transmission [9]. In cross-layer based routing strategy, the WSN permit all the layers to accomplish a data transmission [10].

The performance of sensor networks can be enhanced by rectifying the routing issues related to energy conservation. The cross-layered architecture can help to boost network performance. In this type of architecture, all the layers of the protocol involved to send the network status and transmit information. These types of methodologies can determine a better path for routing with the consideration of both energy consumption and performance of the network [11]. In the cross-layer routing protocol, the different layers of network protocol is 
fused to attain the maximum QoS [12]. Non-hierarchical data exchange between various layers takes place in cross-layer based routing architecture [13]. The analysis founds that the crosslayer based routing can reduce the overhand, also it can optimize the network to achieve the desired QoS [14].

In energy-aware routing of sensor networks, the mechanisms should limit the usage of the number of nodes in the communication path. Thus it is essential to determine a proper path between the particular sensor node and base node [15]. Hence in a later period, the energyaware routing mechanisms are developed with one or more following constraints, such as Node deployment, global addressing, memory \& power constraints and IP based routing [16]. On the other hand, researchers have also tried to minimize the energy consumption in WSN routing by data fusion, MAC optimization, optimal path selection and cross-layer optimization [17]. These WSN routing mechanisms are categorised under one of the three types such as proactive, reactive and hybrid routing. In proactive routing, the routes are predefined without bothering the necessity. But in reactive routing, the routes are defined based on the demand or request. Finally, in the case of hybrid routing, the features of both proactive and reactive routings are combined based on the application. In addition to these mechanisms, hierarchical, location-based and flat-based routing are some of the noticeable strategies for routing in WSN [18].

Transmission time and power consumption are considered as important factors for forming a routing strategy in the WSN. Thus these factors are considered to formulate the mixedinteger non-convex optimization problem. Then the multilevel primal and dual decomposition approach was used to solve the formulated optimization problem [19]. The earlier routing strategies are configured to determine only one path between the sensor and the sink [20]. But the recent strategies determine more than one path to ensure the reliability and robustness of the sensor network [21]. Similarly, by solving a linear programming problem, the optimal operation point based on the parameters like the scheduling, routing and sampling rate can achieve.

Linear programming can solve the problems based on the system needs [22]. The most possible performance is optimized by updating the routing $\&$ scheduling of the system as well as the sampling rate of control systems [23]. In this sense, a bio-inspired algorithm based routing strategy is proposed for WSN and targeted to enhance the performance in terms of energy, reliability and robustness. The major contribution of this paper is, an energy-efficient routing strategy for WSN assisted IoT with better reliability using a hybrid bio-inspired algorithm. The proposed routing strategy includes two major phases such as;

- The nodes are deployed in the grid form based on the grid-based routing strategy. Then a fuzzy logic is employed to determine $\mathrm{CH}$ for every cell in the grid. The fuzzy determines the CH based on Sink Distance (SD), Node Degree (ND), and Residual Energy (RE) [24, 25].

- The hybrid bio-inspired algorithm is proposed to determine the optimal path between, the sensor node to $\mathrm{CH}$ and $\mathrm{CH}$ to sink. Here the moth search and salp swarm optimization techniques are combined to make a hybrid bio-inspired algorithm.

Effective communication can be achieved through cross-layer routing strategies, however energy is a major constraint in WSN assisted IoT. Cluster-based communication is one of the most used strategies for effectively preserving energy in WSN routing. Hence, it is necessary to improve the routing process for better energy preservation. The rest of the paper is organized as follows; some of the studies related to this works are given in section 2 . The proposed 
protocol and effective layer routing technique is shown in sections $3 \& 4$ respectively. The planned proposal results are presented in section 5, and finally, the overall performance is concluded in section 6 .

\section{Related Work}

Some of the recent research works related to the cross-layer routing protocol which is energyefficient in wireless sensor networks (WSNs) are discussed.

Pandey and Agrawal [26] have presented a novel approach towards WSNs which uses an ED-Based Heightened Energy Effective Cross-Layer Model. By utilizing cross-network methodologies, the work seeks to improve performance within a network, which use the crosslayer approach. Alteration of transmission power is done by using a power control technique and this results in a notable energy consumption reduction. The "ED" of each node is considered while calculating weights. This contributes to a significant reduction in control overhead. The results obtained by incorporating ED was compared with other popular algorithms and an effective increase in performance is noted.

An Energy-Efficient Cross-Layer Approach was utilized for Cloud Wireless Green Communications, and it was introduced by Sadiq et al. [27]. Energy-Efficient Cross-Layer (EECL) methodology has been considered which was proposed by using the interaction of the physical and MAC layer information to be used by a network layer to attain effective communication. Evaluation of effectiveness by considering factors like data rate and power utilization was done using MATLAB.

Munawwar et al. [28], designed a novel approach performance analysis of Energy Efficient Cross-Layer Load Balancing, which is utilized in Tactical Multi-gateway Wireless Sensor Network. There were four parameters stimulated and investigated namely Delay, Energy fairness, Packet loss rate and routing overhead. Out of the four modes, normal compression mode makes the best choice for data transmission with having less delay, good energy fairness, less packet loss rate and less routing overhead.

A rule-based clustering for IoT system with consideration of energy was presented by Preeth et al. [29]. A cross-layer optimization system with clustering was presented to stabilize energy consumption. An immune-inspired routing protocol, which used the cross-layer optimization model for routing in IoT. They evaluated the results of the protocol based on the QoS parameters. They have achieved at 100 nodes the average energy consumed was $45 \mathrm{~J}$, network life is 5500 rounds also the packet delivery ratio, end to end delay, and throughput are $99 \%, 2.2 \mathrm{sec}$, and $0.95 \mathrm{Mbps}$ respectively. The same researchers have improved their performance in their further research [30].

Ammar et al. [31] proposed a Cross-Layer approach based on energy minimization for WSN. The energy efficiency of the system was tried to enhance using small-scale channel fading. It was observed that the usage of the cross-layer method extends the lifetime of the network considerably and guarantees effective communication. Their approach has produced a maximum of $99 \%$ packet delivery ratio and $0.03 \mathrm{sec}$ delay.

Dattatraya \& Rao [32] have developed a routing model to enhance the network life and energy efficiency by selecting a suitable cluster head. The authors in this work have utilized a Glow Worm Swarm with Fruit fly algorithm for optimally selecting the most suitable cluster head in WSN. They showed that the considered algorithm requires less iteration and provided better routing performances in terms of cost function. The mean normalized energy was 0.223 and the delay while using 100 nodes is $0.7135 \mathrm{sec}$. 
Amuthan \& Arulmurugan [33] have presented a hyper-exponential reliability factor-based cluster head election to select the most trusted cluster head for communication. To increase the lifetime of the network, the Semi-Markov scheme has been used. They have evaluated the performance of the $\mathrm{CH}$ selection method based on network life and energy efficiency. Here the presented technique has consumed $6.5 \mathrm{~J}$ energy at 100 nodes and the delay was around $0.3 \mathrm{sec}$.

An energy-efficient cluster head selection protocol by Jesudurai \& Senthilkumar [34] to improve the battery performance of the sensor node. This technique also provided improved performances in terms of throughput, energy consumption and network lifetime. Here the maximum energy consumed at 70 nodes was $0.26 \mathrm{~J}$.

Janakiraman [35] have combined the ant colony and artificial bee colony algorithm to effectively select the $\mathrm{CH}$ in WSN. This hybrid algorithm has motivated to properly use the nodes, thus the network performance was improved concerning number of alive nodes, dead nodes, throughput and residual energy. Behera et al. [36] also proposed a $\mathrm{CH}$ selection technique by rotating the position of the cluster heads with higher energy levels. Their $\mathrm{CH}$ selection mechanism has improved the lifetime by $66 \%$, residual energy by $64 \%$ and throughput by $60 \%$.

Lin \& Wang [37] have proposed a dual cluster head mechanism based on clustering to balance the energy consumption in WSN. They have used the game model to balance the consumption of energy by both cluster heads. The performance of the system was evaluated based on energy efficiency. El Alami \& Najid [38] have proposed a hierarchy clustering approach for efficient data communication in WSN. A hierarchical protocol was implemented to identify the nodes with high energy for collecting and transmitting data to the base station. This technique was evaluated in both heterogeneous and homogeneous network to analyze its effectiveness.

The security issues in the IoT have been discussed and solved through deep learning in Lv et al. [39]. A hierarchical intrusion detection model Stacked De-noising Automotive Supporting Vector Machine (SDAE-SVM) has been developed on a three-layer neural autoencoder network to investigate the implementation benefit of deep learning de-noise autoencoder for the internet of object (IoT) fusion protection. The implementation has obtained an accuracy of $97,83 \%$, FPR and FNR of $3.2 \%$ and $1.27 \%$ respectively for a fourlayer network framework, which is significantly better than the existing approaches. Hence, when SDAE is applied, the detection accuracy is increased and is more reliable for intrusion detection based on IoT convergence.

Security for virtual reality has also been implemented by Lv et al. [40] by using an improved version of SVM known as Class and Sample Weighted CSCVM (CSWC-SVM). The data used here is the KDDCUP dataset which contains well-known attacks in the network. The obtained error rate of the proposed approach is lower than the existing C-SVM and RSSVM, while the accuracy is considerably higher. The proposed algorithm has performed well in response to the industrial based network attack, which provides significance for the application of VR in industrial monitoring. Similar security for VR has also been studied by the same researcher [41].

The Access Control Four Store (AC4E) IoT model has been implemented by Lv and Song [42] to examine security and privacy issues for Mobile IoT, by adopting data physical fusion technology. The findings demonstrate that AC4E has good reactivity, accuracy, safety, parallel processing and other features for the analysis of the optimal response path, confidence mechanism and safety speed. AC4E can be utilized to isolate attack node influence and reducing the system energy loss. Further analysis of its secrecy rate shows that nodes in 
different positions can maximise the system's secrecy percentage by a difference in power distribution when the number of iterations is 5 . Thus, the mobile IoT can considerably enhance the network's safety and privacy and provide a specific experimental reference for later enhancement in mobile IoT performance that is of significant investigational importance.

Other researchers have researched cross layer protocol and cluster head selection. The authors in [26-31] concentrated on cross layer mechanism for WSN routing, in these techniques they have given more concentration on energy consumption. In [29], an optimization was tried to perform cross-layer routing mechanism. Then the $\mathrm{CH}$ selection technique was presented in [32-37] by considering the energy consumption as a major constrain, and techniques in [35], [36] evaluated the throughput to ensure the QoS. A clusterbased routing approach similar to LEACH was proposed [38] to reduce energy consumption. All these techniques considered energy consumption as a major constraint, but most of these techniques processed clustering to determine a $\mathrm{CH}$ for transferring data to sink or base station. But these techniques not bothering about the distance between the $\mathrm{CH}$ and sink. In a sensor network, there should be a proper routing mechanism to transfer data from $\mathrm{CH}$ to sink to increase the lifespan of the $\mathrm{CH}$ and network cycle. Thus in this proposed work, more focus is given on determining an effective path between $\mathrm{CH}$ and sink for routing using cross layer mechanism.

\section{Methodology}

An energy preserved Bio-inspired Hybrid Cross-Layer Routing (BiHCLR) strategy for the WSN assisted IoT is presented, in this paper. Here the cross-layer routing is achieved by involving the transport layer, data link layer, and physical layer. The proposed routing strategy is executed in two phases, such as fuzzy-based cluster head $(\mathrm{CH})$ selection and hybrid bioinspired algorithm for path selection. Initially, the fuzzy logic approach is executed for the selection of $\mathrm{CH}$ using the grid-based routing strategy. After the deployment of nodes in the WSN, the fuzzy logic is executed to assign $\mathrm{CH}$, based on the SD, ND and RE. Then the optimal path for routing is selected based on the hybrid bio-inspired algorithm. In this hybrid algorithm, the moth search algorithm and Salp swarm algorithm are combined for optimal routing.

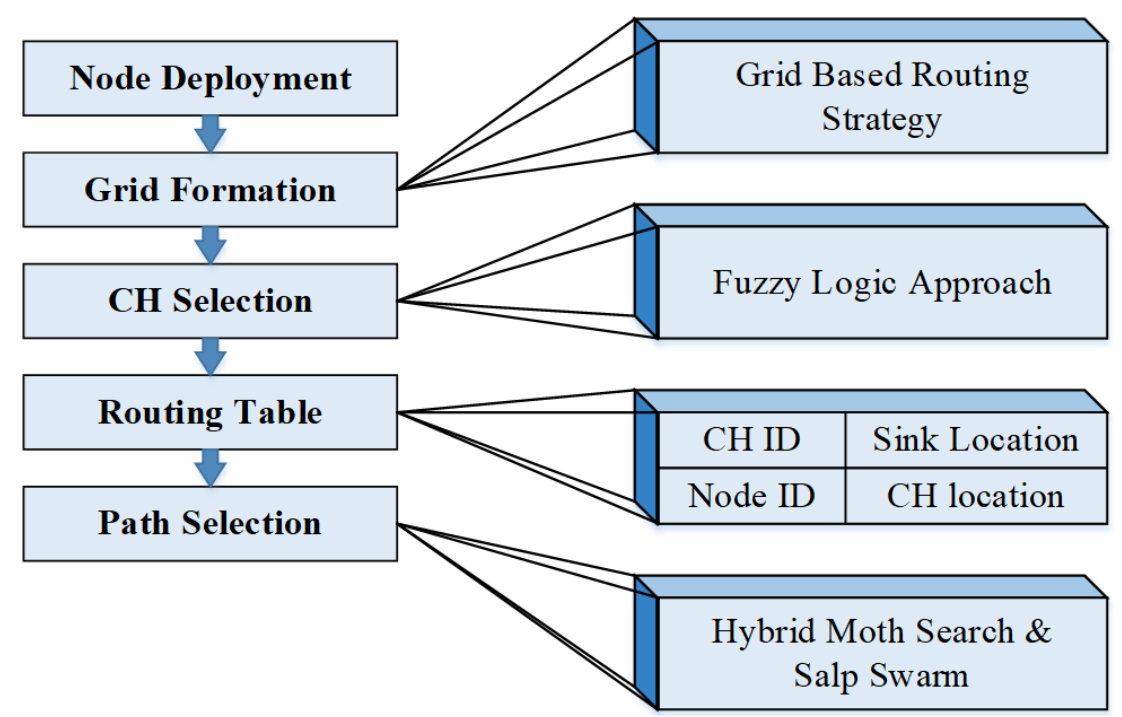

Fig. 1. Architecture of the BiHCLR 
The architecture of the proposed BiHCLR is shown in Fig. 1, for the energy-aware routing in the WSN assisted IoT. Initially, the nodes in the network are gathered in the form of a grid, based on the grid-based routing algorithm. Then one of the nodes from every grid cell is selected as $\mathrm{CH}$ and a most effective node (energy-based) of the network is assigned as a sink node. Subsequently, the hybrid bio-inspired algorithm is used to determine the routing path.

The grid-based routing algorithm includes two major models such as Network model as well as the Energy model. In WSN, initially, the grid is formed then the sensor nodes are placed in every grid, and finally a sink node will be placed in any one of the grids. The grid is a two-dimensional matrix as shown in Fig. 2. The grid region is specified as a square shape and the size of the network is based on the transmission radio range. Fig. 3 shows the representation of grid cell identification in WSN.

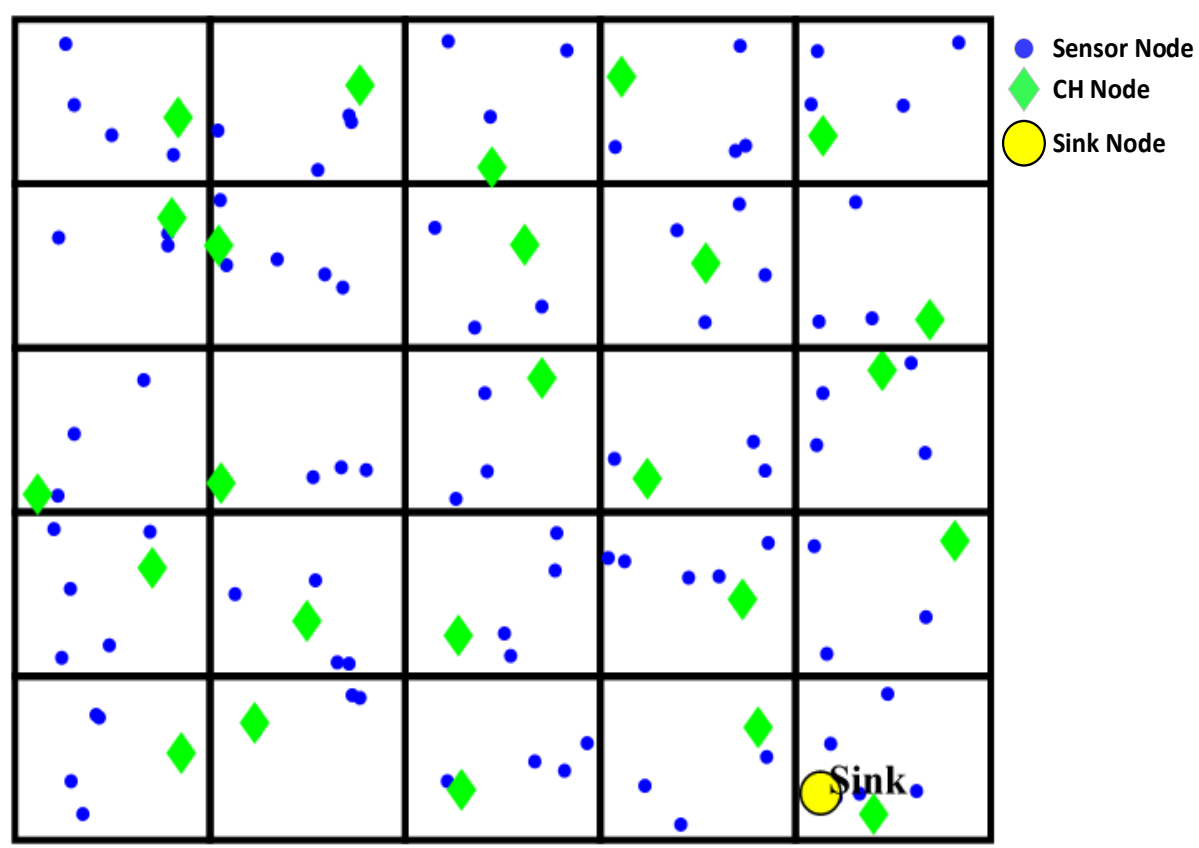

Fig. 2. Grid based sensor node deployment in WSN

\begin{tabular}{|c|c|c|c|}
\hline$[\mathbf{a}, \mathbf{a}]$ & {$[\mathbf{a}, \mathbf{b}]$} & {$[\mathbf{a}, \mathbf{c}]$} & {$[\mathbf{a}, \mathbf{d}]$} \\
\hline$[b, a]$ & {$[\mathbf{b}, \mathbf{b}]$} & {$[\mathbf{b}, \mathbf{c}]$} & {$[\mathbf{b}, \mathbf{d}$} \\
\hline$[\mathbf{c}, \mathbf{a}]$ & {$[c, b]$} & {$[\mathbf{c}, \mathbf{c}]$} & {$[\mathrm{c}, \mathrm{d}]$} \\
\hline $\mathbf{d}, \mathbf{a}]$ & {$[\mathbf{d}, \mathbf{b}]$} & {$[\mathbf{d}, \mathbf{c}]$} & {$[\mathrm{d}, \mathrm{d}$} \\
\hline$[\mathbf{e}, \mathbf{a}]$ & {$[\mathbf{e}, \mathbf{b}]$} & {$[\mathbf{e}, \mathbf{c}]$} & {$[\mathbf{e}, \mathbf{d}]$} \\
\hline
\end{tabular}

Fig. 3. Representation of grid cell identification in sensor network Grid 


\subsection{Network Model}

In this type of model, the initial sensor nodes are arranged in grid form. This model focuses on cluster formation and cluster head selection. The network region is modelled as a square field with uniformly distributed sensor nodes. Sensor nodes collect data from the sensing field and forward the same data to the base station. It is considered that all the sensor nodes have the same configuration and limited energy. The nodes are randomly deployed inside the grid, and the BS is stationary and placed inside or outside the sensing region. The grid-based energyefficient routing algorithm consists of three phases which are grid formation, grid coordinator selection and data transmission.

\subsection{Energy Model}

The radio energy model can determine the energy consumption in the sensor network. In proportion to this model, a message of size one bit is sent to the sender $\mathrm{x}$ to the neighbourhood $\mathrm{y}$, and the consumed energy is represented as $\mathrm{e}_{(\mathrm{x}, \mathrm{y})}$ and the distance is represented by $d(x, y)$. $E_{x}$ is the amount of required energy to perform for the signal transmission from $\mathrm{x}$ to $\mathrm{y}$. Here, $E_{r}$ is the required energy. The energy consumption is computed using (1).

$$
e(x, y)=e_{1}+e_{2} * d(x, y) * \alpha
$$

where $\alpha$ is the path loss constant and $e_{1}, e_{2}$ are constants that depend upon the WSN devices.

Each cell in the grid is represented by a unique identifier in the grid as shown in Fig. 3. The energy model is a simple class and its principal purpose is to track the condition of the network and to measure its energy usage accordingly. It manages the energy source notification when node energy is exhausted. It also keeps a record of the device's overall energy usage. Hence, this energy consumption is measured and used for in the optimization of the $\mathrm{CH}$. In this model to attain reliable operation, considered the transmission range depends upon the sensitivity, grid size and transmission power of the sensor nodes. The sensor nodes are set to directly communicate between grids in the single hop. The transmission radio or signal scale is denoted as ' $R$ ', and the size of the grid is indicated as ' $a$ ' [43].

$$
\begin{gathered}
R=a \sqrt{2}+a \sqrt{2} \\
a=\frac{R}{2.83}
\end{gathered}
$$

Then the selected $\mathrm{CHs}$ are ranked based on degree calculation, which is the ratio of number of nodes in the cell to the total nodes in the network. The Cluster head degree is calculated using (4). It is the ratio of the number of nodes in the cluster to the total number of nodes.

$$
C_{\text {Degree }}=\frac{\text { Number of } \text { Nodes in the cluster }}{\text { Total Number of Nodes }}
$$

The $\mathrm{CH}$ degree helps to evaluate the capacity of the grid cell based on the ranking. That is higher the degree value represents the lower the acceptance rate of a new node. For an effective grid-based communication, the $\mathrm{CH}$ degree should be even or its variance should be less to maintain reliable communication in WSN. Based on the above equations, fuzzy logic is used to select the cluster heads as detailed in the next section. 


\subsection{CH Selection using Fuzzy Logic Approach}

The deployed nodes are arranged in form of grid on grid-based routing strategy. Then the fuzzy logic is executed to select the $\mathrm{CH}$ for every cell in the grid based on SD, ND and RE. The layer routing is performed in a grid by grid manner, and the sensor nodes are randomly deployed [43]. The role of the cluster head is to transmit the data to the BS by forwarding the messages through the neighbouring cluster heads.

In each grid, the sensor nodes are denoted as grid members. The cluster members balance the load in a cluster, and one of the sensor nodes is selected as a cluster head. In the grid, the cluster heads are selected by using the fuzzy decision-making system. The proposed method has three inputs, which are residual energy of the cluster head, node degree, and the distance between cluster head and sink, while the output is the node that is selected as the cluster head.

In the fuzzification stage, the values in the grid are converted into linguistic variables. These linguistic variables used as membership functions in the fuzzy. The membership functions assigned for each input variable is shown below:

- Distance between CH and BS - Near (N), Medium (M), and Far (F).

- $\quad$ Residual energy of $\mathrm{CH}$ - High (H), Medium (M), and Low (L).

- $\quad \mathrm{CH}$ degree - Low (L), Average (A), and High (H).

Similarly, for the output variable, lots of membership functions are used as given below:

- $\quad$ Selection of Cluster heads - Very High (VH), High (H), Medium High (MH), Ultra High (UH), Medium (M), Medium Low (ML), Low (L) and Very Low (VL).

These input and output membership functions are shown in Table 1.

Table 1. Membership Functions

\begin{tabular}{|c|c|c|c|c|c|}
\hline \multirow{3}{*}{ I/P } & Distance between CH and BS & N & \multicolumn{2}{|c|}{ M } & F \\
\cline { 2 - 6 } & Residual Energy of CH & L & \multicolumn{2}{|c|}{ M } & H \\
\cline { 2 - 6 } & Degree of CH & L & \multicolumn{2}{|c|}{ A } & H \\
\hline \multirow{2}{*}{ O/P } & Selection of CH & VL & L & ML & M \\
\cline { 2 - 6 } & & UH & MH & H & VH \\
\hline
\end{tabular}

The membership function for the fuzzy rule is assigned triangular and trapezoidal member functions as shown in (5) and (6). The triangular curve contains both scalar and vector functions, where the vector function is denoted by a, which depends on scalar parameters, $1_{1}$, $\mathrm{m}_{1}$, and $\mathrm{n}_{1}$, as given in (5).

$$
\mu_{Q 1}(a)=\left\{\begin{array}{cc}
0 & a \leq l_{1} \\
\frac{a-l_{1}}{m_{1}-l_{1}} & l_{1} \leq a \leq m_{1} \\
\frac{a-m_{1}}{n_{1}-m_{1}} & m_{1} \leq a \leq n_{1} \\
0 & n_{1} \leq a
\end{array}\right.
$$


Where, $a$ is the member of the universal set, $l_{1}$ is the lower boundary, $n_{1}$ is the higher boundary and $m_{1}$ represent the middle point of the triangle. While the triangular function has three scalar parameters, the trapezoidal function has four scalar parameters denoted by $l_{2}, m_{2}$, $n_{2}$, and $o_{2}$.

$$
\mu_{Q 1}(a)=\left\{\begin{array}{cc}
0 & a \leq l_{2} \\
\frac{a-l_{2}}{m_{2}-l_{2}} & l_{2} \leq a \leq m_{2} \\
1 & m_{2} \leq a \leq n_{2} \\
\frac{o_{2}-a}{o_{2}-n_{2}} & n_{2} \leq a \leq o_{2} \\
0 & a>o_{2}
\end{array}\right.
$$

Where, $l_{2}$ is the lower limit, $m_{2}$ is the lower support limit, $o_{2}$ is the upper limit of the trapezoidal, $n_{2}$ upper support limit. The proposed system uses the Mamdani fuzzy inference rule. Two rules are used for the approach as shown in Table 2 which are

- Rule1: If (RE is $\mathrm{L}$ ) and ( $\mathrm{SD}$ is $\mathrm{F})$, then ( $\mathrm{CH}$ is $\mathrm{VL}$ )

- Rule2: If (RE is $\mathrm{H})$ and ( $\mathrm{SD}$ is $\mathrm{N})$, then ( $\mathrm{CH}$ is $\mathrm{VH})$

Table 2. Fuzzy Rules

\begin{tabular}{|c|c|c|c|c|c|c|c|c|c|c|c|c|c|}
\hline RE & SD & ND & $\begin{array}{c}\text { CH } \\
\text { selection }\end{array}$ & & RE & SD & ND & $\begin{array}{c}\text { CH } \\
\text { selection }\end{array}$ & RE & SD & ND & $\begin{array}{c}\text { CH } \\
\text { selection }\end{array}$ \\
\hline H & N & L & VH & & M & N & L & H & & L & N & L & H \\
\hline H & N & M & H & & M & N & M & M & & L & N & M & M \\
\hline H & N & H & M & & M & N & H & M & & L & N & H & L \\
\hline H & M & L & H & M & M & L & H & & L & M & L & M \\
\hline H & M & M & M & & M & M & M & L & & L & M & M & M \\
\hline H & M & H & L & & M & M & H & L & & L & M & H & VL \\
\hline H & F & L & M & M & F & L & M & & L & F & L & L \\
\hline H & F & M & L & M & F & M & L & & L & F & M & M \\
\hline H & F & H & L & & M & F & H & L & & L & F & H & VL \\
\hline
\end{tabular}

Then at the output phase, the defuzzification is performed in which the predicted membership functions are converted into crisp (binary) values using (7).

$$
Z=\frac{\sum_{l=1}^{q} a_{i} \mu\left(a_{i}\right)}{\sum_{i=1}^{q} \mu\left(a_{i}\right)}
$$

Where, $a_{i}$ epitomizes each representative of the output world, $\mu a_{i}$ indicates the association degree of $a_{i}$ and $q$ denotes the number of representatives in output. 


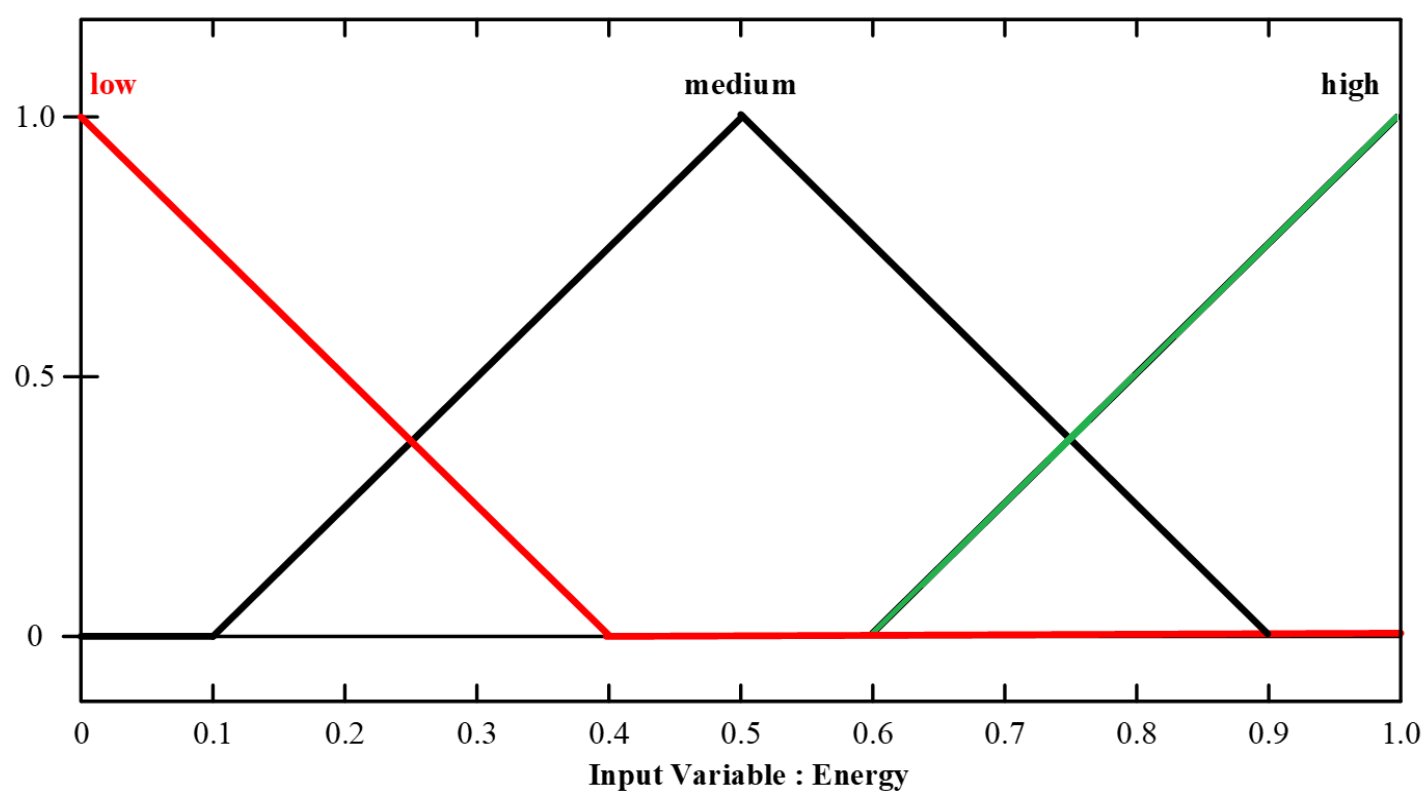

Fig. 4. Membership functions of residual energy in $\mathrm{CHs}$

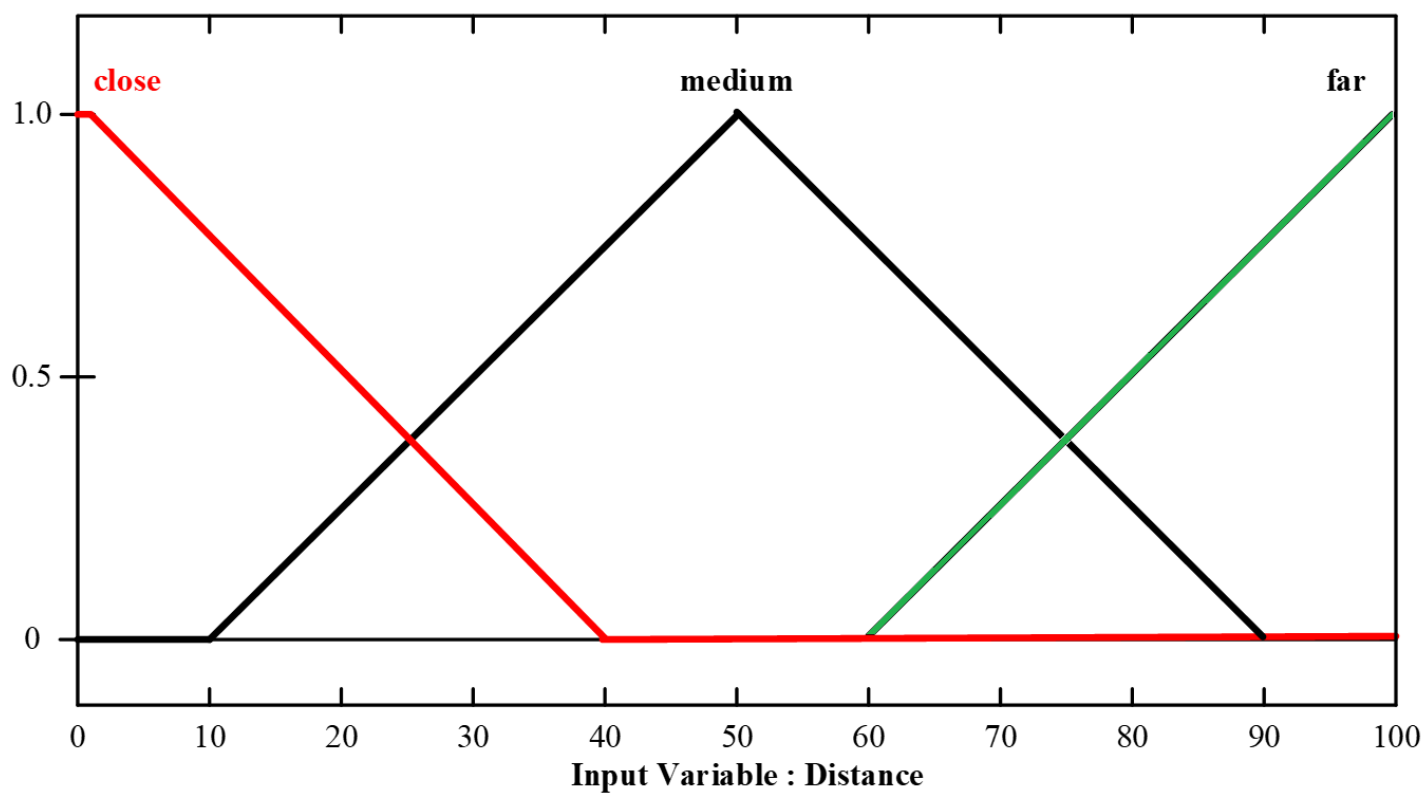

Fig. 5. Membership functions of sink distance

Fig. 4 and Fig. 5 shows the membership function of residual energy and sink distance respectively. The fuzzy rules are assigned in the membership function, and the output is extracted by applying the rules. These fuzzy rules are then applied to the optimization approach for routing as shown in the next section.

\subsection{Hybrid Bio-Inspired Algorithm for Routing}

An intelligent cluster head selection is performed using a grid-based method with a fuzzy logic 
system. The optimal path for routing is determined using a Bio-inspired algorithm. In this work, a hybrid approach that combines the Moth search algorithm and Salp swarm algorithm is used for the optimization and to determine the better routing path. The bio-inspired algorithm includes three main phases, which are explained below.

\subsubsection{Initialization}

In this phase, the available paths between $\mathrm{CH}$ and sink nodes are initialized as initial population for the optimization. Initially, one of the $\mathrm{CHs}$ is considered as the sender and all other $\mathrm{CHs}$ are considered as intermediate paths or sink. Thus in this initialization phase all the possible paths between $\mathrm{CH}$ and sink, which is represented in (8).

$$
\text { Sol }=P_{i}, i=1,2, \ldots N \text {. }
$$

where, 'Sol' represents the initial population set, ' $P_{i}$ ' indicates the $\mathrm{i}^{\text {th }}$ path between $\mathrm{CH}$ and sink, and ' $N$ ' is the total number of paths. The path includes distance and total energy.

$$
P=\{R E, D I S T\}
$$

where 'RE' is the residual energy of nodes in the path, 'DIST' is the total distance of the path. The standard deviation for residual energy $\left(\sigma_{R E}\right)$ is used for the measurement of the quality of uniform load distribution among sensor nodes and is given in (9).

$$
R E=f_{1}=\sigma_{R E}=\sqrt{\frac{1}{n}} \sum_{j=1}^{n}\left\{\mu_{R E}-e\left(\operatorname{node}_{j}\right)\right\}^{2}
$$

Here,

$$
\mu_{R E}=\frac{1}{n} \sum_{i=1}^{n} E\left(\text { node }_{i}\right)
$$

where, ' $n$ ' is the total number of nodes that exist in the path and ' $E\left(\right.$ node $\left._{j}\right)$ ' is the residual energy of $\mathrm{i}^{\text {th }}$ node in the path. Then the distance between the sender $\mathrm{CH}$ to the sink is calculated as the sum of Euclidean distance between each $\mathrm{CH}$ in the path, which is given in (11).

$$
D I S T=\sum_{i}^{n-1} \sqrt{\left(C_{i}(x)-C H_{i+1}(x)\right)+\left(C_{i}(y)-C H_{i+1}(y)\right)}
$$

where, ' $\mathrm{CH}_{i}(x)$ ' and ' $\mathrm{CH}(\mathrm{H})$ ' represents the $\mathrm{x}$ and $\mathrm{y}$ co-ordinate of $\mathrm{i}^{\text {th }} \mathrm{CH}$ in the path respectively. Thus the first objective of the proposed optimization is energy reduction which is formulated in (10). The second objective is to reduce the path distance, which is formulated in (11). Hence these two parameters of every path are initialized as the initial population.

\subsubsection{Fitness Evaluation}

In this phase, the fitness of every solution or path between the $\mathrm{CH}$ and sink is calculated. The major objective of the optimization is to find the path with less energy consumption and short distance. Thus, the formulated objective function includes both the energy and distance of every path. The fitness function is formulated as the minimization function and it is the product 
of energy and distance, which is given in (12).

$$
\mathrm{F}_{i}=\min \left\{\mathrm{RE}_{i} \times \mathrm{DIST}_{i}\right\}
$$

where, ' $\mathrm{F}_{i}$ ' is the fitness of $\mathrm{i}^{\text {th }}$ population, ' $\mathrm{RE}_{i}$ ' is the energy required in the $\mathrm{i}^{\text {th }}$ population and 'DIST $i$ ' is the total distance of $\mathrm{i}^{\text {th }}$ path or population.

\subsubsection{Solution Update and Path Selection}

In this phase, based on the fitness value a path is selected or it is used to update the solution. Here the Moth search and Salp Swarm Optimization is combined to execute the hybrid bioinspired optimization. The Moth Search (MS) optimization is one of the recently developed population-based algorithms that mimic the navigation methods of a moth. Generally, moths fly around the light source mistaking it as the moon [44, 45]. The position of Moth is considered to solve the optimization problem.

One the other hand, the Salps are aquatic animals that look like jellyfish and move in groups. They form groups known as Salp chains which have two groups head and followers [46]. They work together in swarms for movement and to identify food sources. The leader Salp is at the front and finds the food, while the follower Salps will follow the leader Salp to reach the food source. This behaviour has inspired an optimization approach that proposes to use the low-level nodes to find a high-level network. The approach is known as Salp Search Algorithm (SSA).

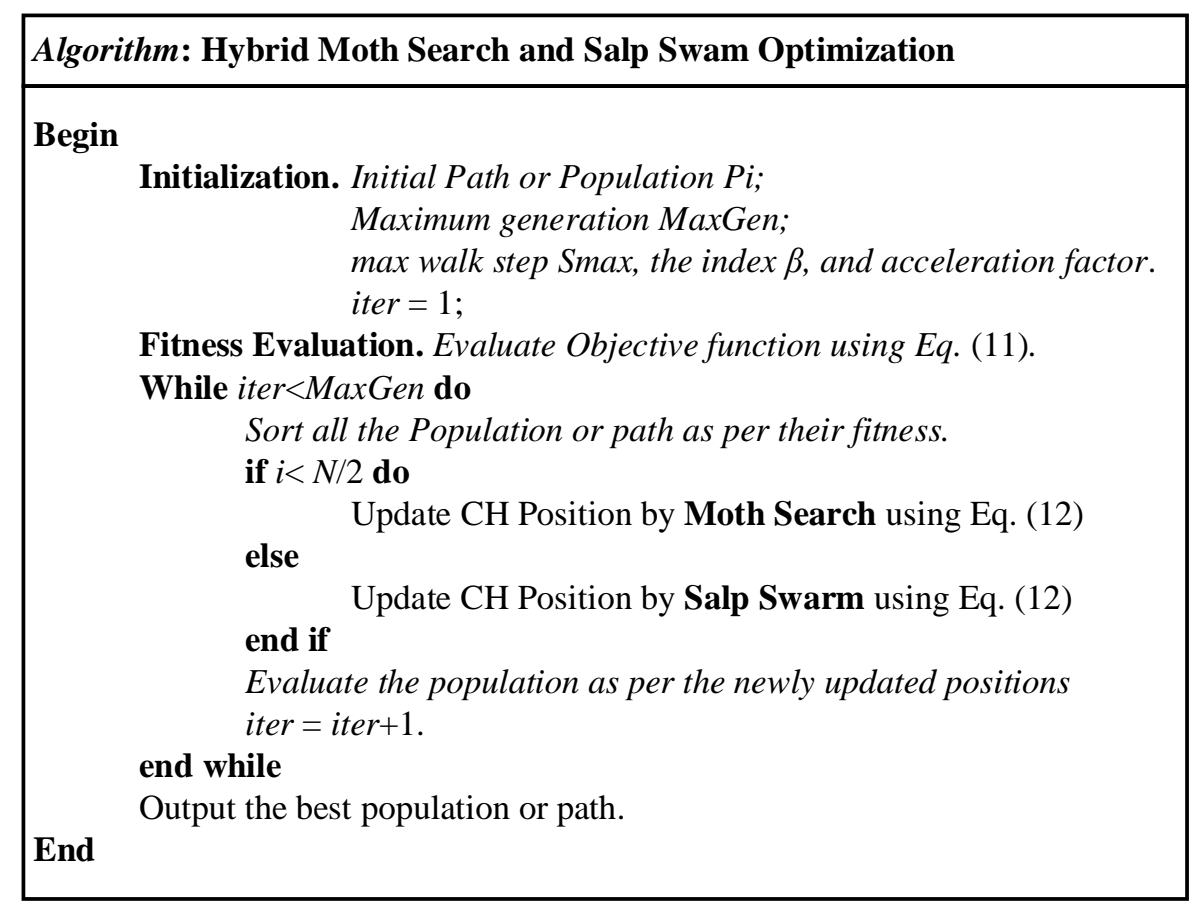

Fig. 6. Pseudocode for Hybrid Bio-inspired algorithm

After the fitness evaluation, all the population are arranged in increasing order, half of the paths or population are updated levy flight as per moth search optimization. Then the remaining populations are updated based on the update rule of the Salp swarm. The 
pseudocode of the hybrid moth salp algorithm is given in Fig. 6. The first 50\% of positions of the cluster heads are updated by performing moth search Levy flight distribution [47]. The last $50 \%$ of positions are updated based on Salp swarm [27] is given in (13).

$$
x_{t}^{i+1}=\left\{\begin{array}{cc}
x_{t}^{i}+\alpha L(s) & i<N / 2 \\
\frac{1}{2}\left(x_{t}^{i+1}+x_{t}^{i}\right) & \text { otherwise }
\end{array}\right.
$$

where, $x_{t}^{i}$ and $x_{t}^{i+1}$ are the original and updated position of $\mathrm{CH}$ respectively at the present generation of population, ' $\mathrm{t}$ ' is the dimension of the population. $L(s)$ is step drawn from Levy flight described as shown in (14),

$$
L(s)=\frac{(\beta-1) \Gamma(\beta-1) \sin (\pi(\beta-1)}{\pi s^{\beta}}
$$

The accelerating factor is the ratio of a rate at a high temperature to a lower temperature. Here, the acceleration factor $\beta$ is selected as 1.5 and assumed that $\mathrm{S}$ is greater than zero. The value 1.5 is randomly selected based on trial and error. The parameter $\alpha$ is denoted as scale factor. It can be formulated as shown in (15).

$$
\alpha=\frac{s_{\max }}{t^{2}}
$$

Here, $\mathrm{S}_{\max }$ is the maximum walk step that will move from one $\mathrm{CH}$ to the other $\mathrm{CH}$. The base station or sink node periodically broadcasts its information such as the present position and node identification (ID) to the network by using the grid-based protocol. The nodes receive the sink messages and update their neighbour lists.

The routing tables are used to assemble the routing path. These nodes use grid rules to connect with the BS. One of the target cluster head is selected in between the grids and the shortest path to the cluster head is identified among them all. Some existing cross-level protocols are introduced to assign co-ordination issues between high level and low-level nodes. But the moth inspired algorithm solves this issue. It can provide the shortest transmission distance to the sink node. The cluster head selection is carried out by selecting the sensor nodes with the residual energy greater than $0.4 \mathrm{~J}$. 


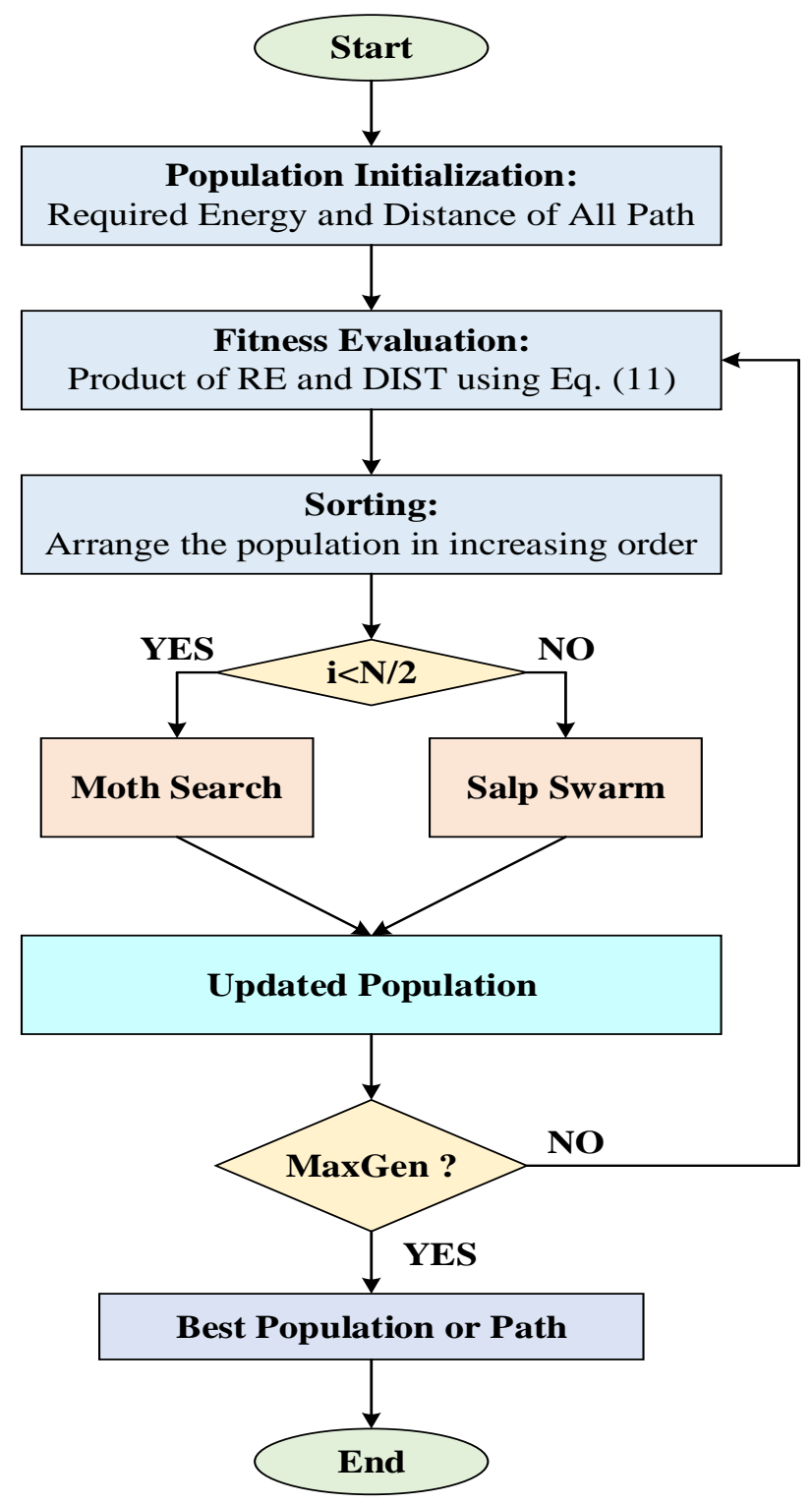

Fig. 7. Flow Diagram of BiHCLR

The flow diagram of the proposed BiHCLR approach is shown in Fig. 7. The optimal value of the transmission range can be adjusted by the MS and SSA algorithm after the number of generations is performed. The iteration is carried out a maximum of 50 times; otherwise, the iteration is not sustained and loops back to (14) to update the $\mathrm{CH}$ position and minimize the transmission range. The performance of the algorithm is better if it obtains the minimum value of the transmission range (R). Every population-based algorithm has two subpopulations which are global best and local best. Once it reaches the defined iterations which in this case is 50 , the process is completed. 


\section{Results and Discussion}

The Bio-inspired cross-layer routing protocol is designed to improve reliability, energy consumption and QoS. Thus, it concentrates on QoS parameters such as network lifetime, energy consumption, bit error rate, throughput, transmission delay, packet loss and buffer occupancy. All these parameters are computed with several nodes. The implementation of this work is carried out using MATLAB. The simulation parameters initialized are shown in Table 3. While the number of nodes vary, the parameters for 100 nodes are tabulated. However, the number of nodes vary throughout the simulation. The parameters of the performance analysis are as follows:

1. Energy Consumption: $30 \mathrm{~mJ}$

2. Network Lifetime: 5600 rounds

3. Throughput: $0.99 \mathrm{Mbps}$

4. Bit Error Rate: $2 \%$

5. Packet Loss: $<0.3 \%$

6. Transmission Delay: $3.2 \mathrm{sec}$

7. Buffer Occupancy: $2 \%$

Table 3. BiHCLR Simulation Parameters

\begin{tabular}{|l|l|}
\hline \multicolumn{1}{|c|}{ Parameters } & \multicolumn{1}{c|}{ Simulation values } \\
\hline Area & $1250 \times 1250 \mathrm{Km}$ \\
\hline Location of BS (sink) & {$[1083 \quad 83]$} \\
\hline Number of Nodes & 500 \\
\hline Number of CHs & 24 \\
\hline Initial Energy & $0.5 \mathrm{~J}$ \\
\hline Packet Size & $512 \mathrm{~Kb}$ \\
\hline Simulation Time & $7.49 \mathrm{~ms}$ \\
\hline Bandwidth & $9 \mathrm{Mbps}$ \\
\hline
\end{tabular}

Table 4. Performance metrics for 500 nodes

\begin{tabular}{|c|c|c|c|c|c|}
\hline Metrics & HEED & NF-EPO & FRDLG & FEEC-IIR & BiHCLR \\
\hline Energy Consumption (mJ) & 250 & 160 & 175 & 149 & 70 \\
\hline Network Lifetime (Rounds) & 3100 & 4100 & 3980 & 4300 & 5250 \\
\hline Throughput (Mbps) & 0.48 & 0.52 & 0.47 & 0.62 & 0.91 \\
\hline Bit Error Rate (\%) & 25 & 21 & 23 & 16 & 8.5 \\
\hline E2E Delay (sec) & 9.5 & 9 & 9.4 & 6.1 & 4.5 \\
\hline Packet Loss Ratio (\%) & 12 & 6 & 5 & 8 & 4 \\
\hline Buffer Occupancy (\%) & 23 & 18 & 20 & 15 & 8.5 \\
\hline Packet Delivery Ratio (\%) & 87 & 94.5 & 92.5 & 95 & 95.7 \\
\hline
\end{tabular}

Table 4 above presents the performance metrics for 500 nodes of the proposed BiHCLR parameters which are compared with existing techniques like NF-EPO (Neuro FuzzyEmperor Penguin Optimization), FEEC-IIR (Adaptive Fuzzy-rule-based Energy Efficient Clustering and Immune-Inspired Routing), FRLDG (Fuzzy Reinforcement Learning-based Data Gathering) and HEED (Hierarchical Energy Efficient Data gathering). The grid-based layer routing for the proposed cross layer routing is shown in Fig. 8. Once the simulation is run, the transmission path occurs through different nodes until it reaches sink. One of the examples of the cross layer routing is shown in the figure. 


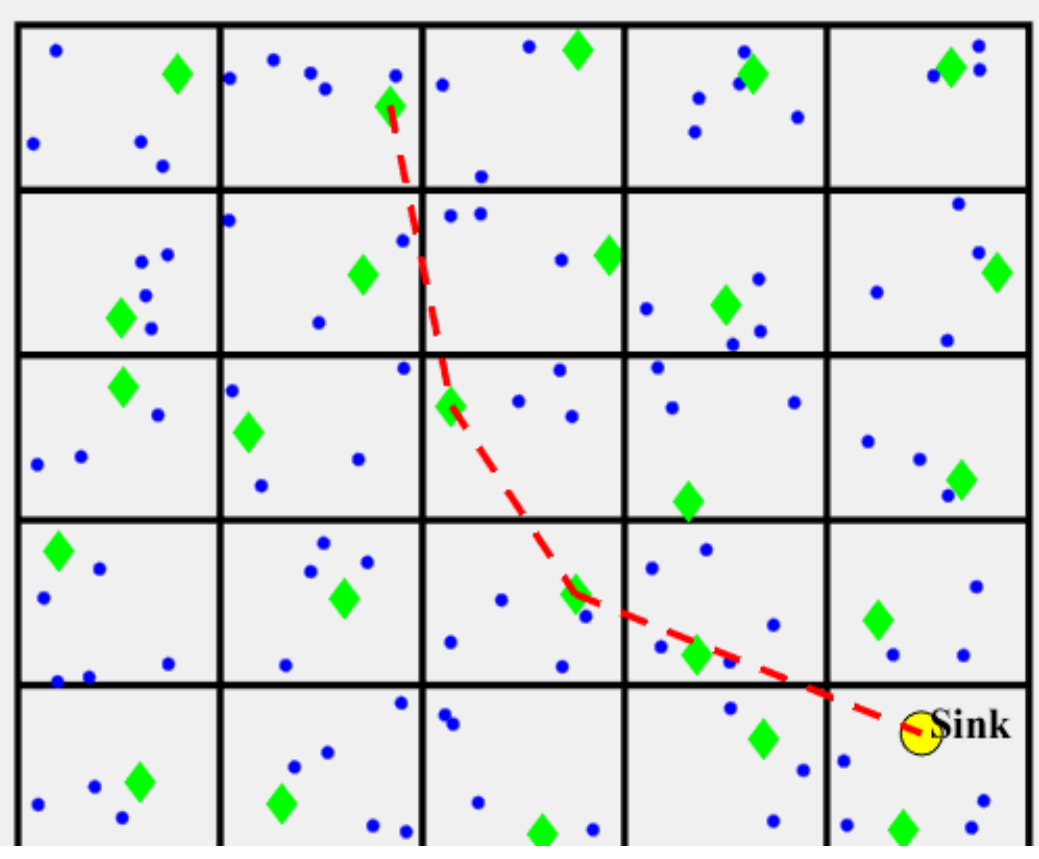

Fig. 8. Implementation of Grid based layer routing

During the routing period, the sensor nodes consume energy. This energy is computed using the sum of receiving energy with a number of nodes and the transmitted energy. The BiHCLR technique monitors the residual energy of the nodes, and if the consumption of energy is less, then the lifetime of the network can be increased. The proposed BiHCLR technique efficiently manages energy as shown in the results below.

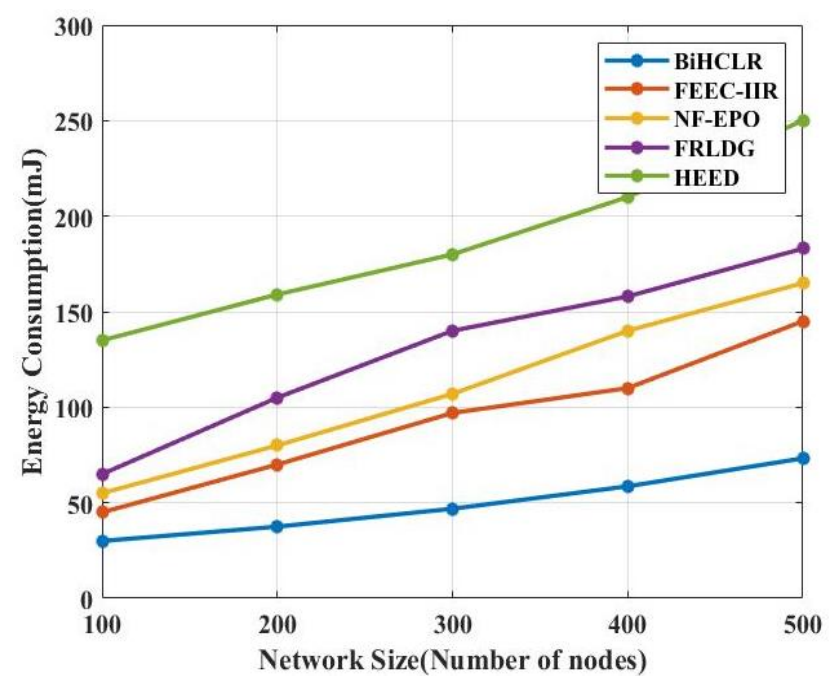

Fig. 9. Network Energy Consumption vs Network Size

Fig. 9 shows the performance of the proposed BiHCLR energy consumption. The BiHCLR use less energy compared to other routing protocols of FEEC-IIR, FRLDG, NF-EPO, and HEED. BiHCLR uses $30 \mathrm{~mJ}$ for 100 nodes, while HEED consumes $142 \mathrm{~mJ}$. The energy 
consumptions of the other methods are FEEC-IIR $(45 \mathrm{~mJ})$, NF-EPO $(55 \mathrm{~mJ})$, and FRLDG $(65 \mathrm{~mJ})$. As the number of nodes increases, energy consumption also increases. For 500 nodes, the proposed approach consumes $70 \mathrm{~mJ}$, while the others have a very high consumption rate of FEEC-IIR (145mJ), NF-EPO (160mJ) FRLDG $(180 \mathrm{~mJ})$ and HEED $(250 \mathrm{~mJ})$.

The lifetime of the network is defined as the operational time of the network during which it can perform the tasks. To achieve an effective lifetime, the network balances the energy consumption and data traffic. When the first node of the network dies, the other nodes lose their energy in a short duration. Fig. 10 shows the comparison of the lifetime of the sensor nodes with the other techniques.

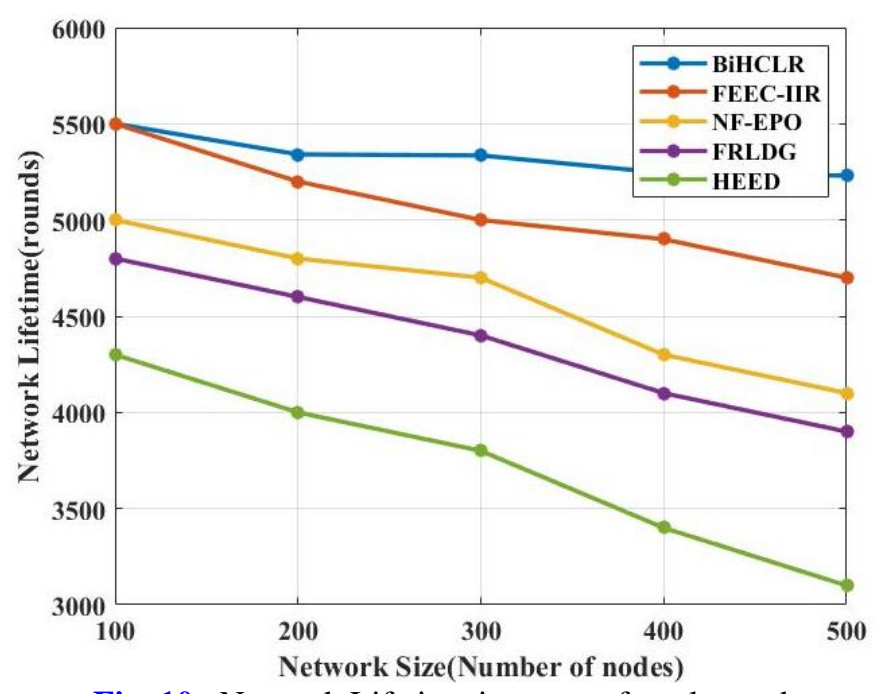

Fig. 10. Network Lifetime in terms of total rounds

The obtained result for the proposed approach has a longer life when compared to other methods. When the number of nodes is increased, the lifetime decreases. It is seen that this reduction is very less in the proposed approach while the lifetime of the other conventional techniques falls drastically with the increase in the number of nodes. The lifetime of the proposed BiHCLR for 100 nodes is 5600 rounds. The lifetime of other methods for 100 nodes for FEEC-IIR, NF-EPO, FRLDG, HEED is 5500 rounds, 5000 rounds, 4800 rounds and 4300 rounds respectively.

The throughput is defined as the ratio of the number of packets received at the receiver to the packet transmission delay in the process. To achieve good throughput, the data needs to be transmitted with good transmission power.

$$
\text { Throughput }=\frac{\text { Number of } \text { Packets Received }}{\text { Transmission Delay }}
$$

Fig. 11 shows the throughput of the proposed approach and has compared it with conventional methods. The maximum throughput is achieved by adjusting the transmission delay. When the number of nodes is increased, the throughput drastically decreases for the other techniques; however, the proposed method has a high throughput even for 500 nodes. The throughput of the proposed BiHCLR is $0.99 \mathrm{Mbps}$ for 100 nodes, while for the other methods it is $0.95 \mathrm{Mbps}$ (FEEC-IIR), 0.90Mbps (NF-EPO), 0.80Mbps (FRLDG) and $0.77 \mathrm{Mbps}$ (HEED). For 500 nodes, the throughput of BiHCLR remains above 0.9Mbps, while it falls below 0.6 for all the other techniques; hence the proposed method has a superior throughput. 

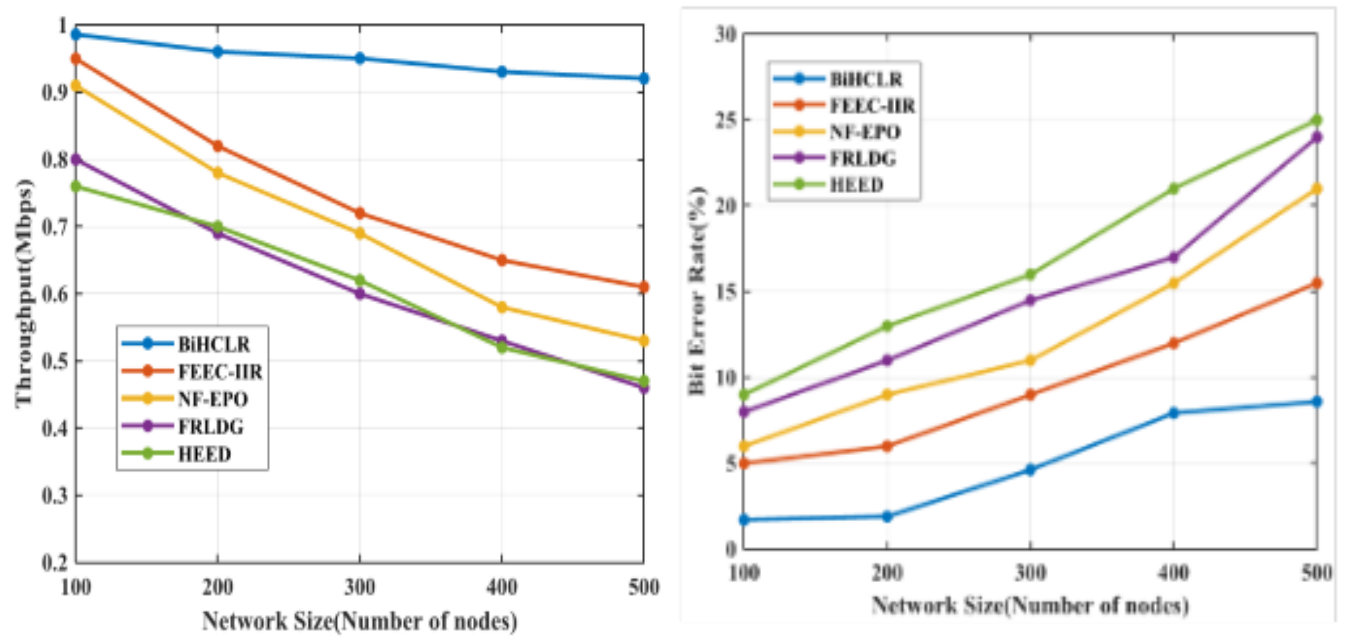

Fig. 11. Performance assessment of Throughput Fig. 12. Performance assessment of bit error rate

The BER value increases with an increase in the number of nodes. When the BER is low, the bandwidth and interference of the data link are also low. The Bit Error Rate (BER) of the proposed BiHCLR is shown in Fig. 12, and it is lower than the compared techniques. Bit error rate of BiHCLR is $2 \%$ and for the existing methods, it is at $5 \%, 6 \%, 8 \%, 9 \%$ respectively for FEEC-IIR, NF-EPO, FRLDG, HEED. Similarly, for 500 nodes, the BER of the proposed method remains below $10 \%$, while it is significantly higher for the other methods.

Packet loss is the number of packets that do not reach the target node within the stipulated time. The packet loss for the proposed method and conventional methods are shown in Fig. 13. There is less than $1 \%$ packet loss ratio for 100 nodes, while it is high for the other methods especially for HEED which has a packet loss of $7 \%$. The packet loss of other methods is FEECIIR (1\%), NF-EPO (2\%), FRLDG (3\%) and HEED (7\%). When the number of nodes increases, the packet loss also increases. For 500 nodes, the loss is $4 \%$, while the packet loss of other methods is FEEC-IIR (5\%), NF-EPO (6\%), FRLDG (8\%) and HEED (12\%).

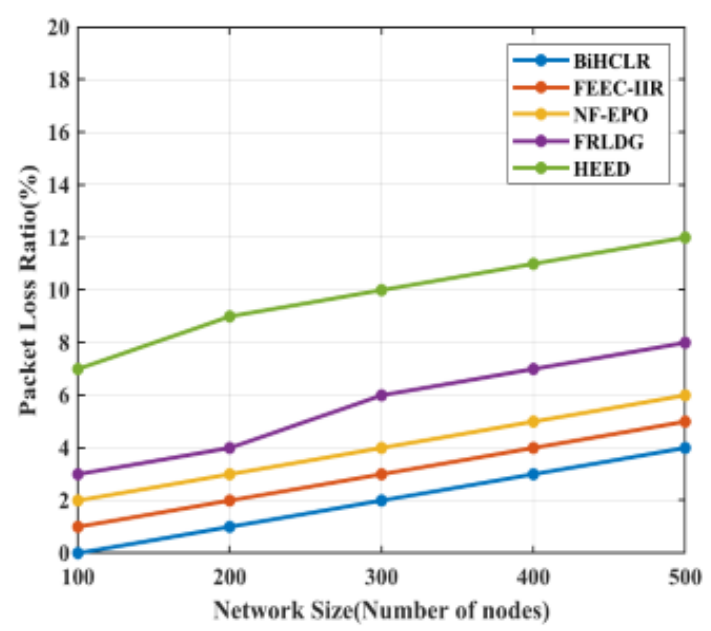

Fig. 13. Performance assessment of packet loss 
The total time required to transmit the packet to a receiver and to packets received by the receiver. Average time consumed in one packet of data transmission in the WSN. The transmission delay is reduced compared to other methods because of our proposed BiHCLR has achieved the shortest path routing. Fig. 14 shows the comparison of end to end delay. The proposed method indicates less transmission delay ( $3.2 \mathrm{sec}$ ) in 100 nodes compared to other existing approaches. When the number of nodes increases, the transmission-delay also increases.

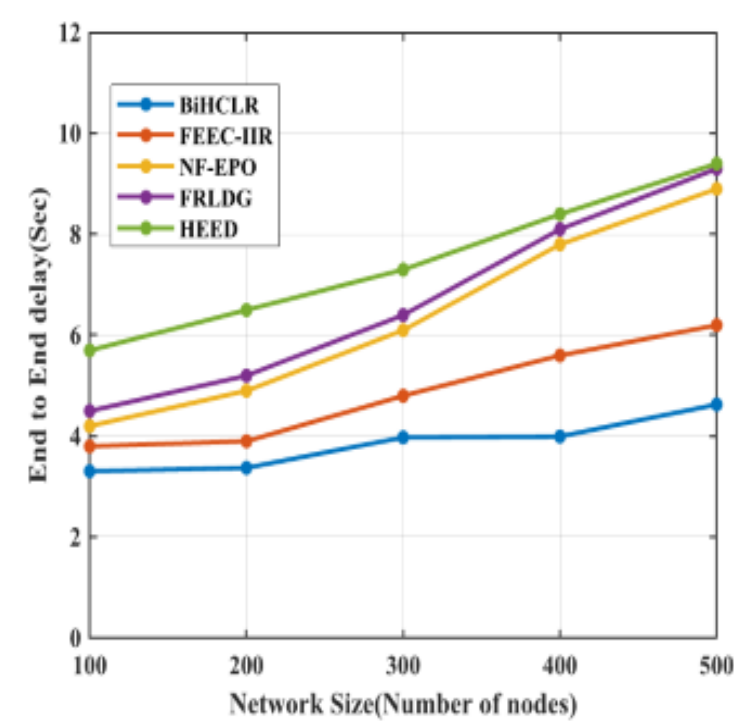

Fig. 14. Performance assessment of E2E delay

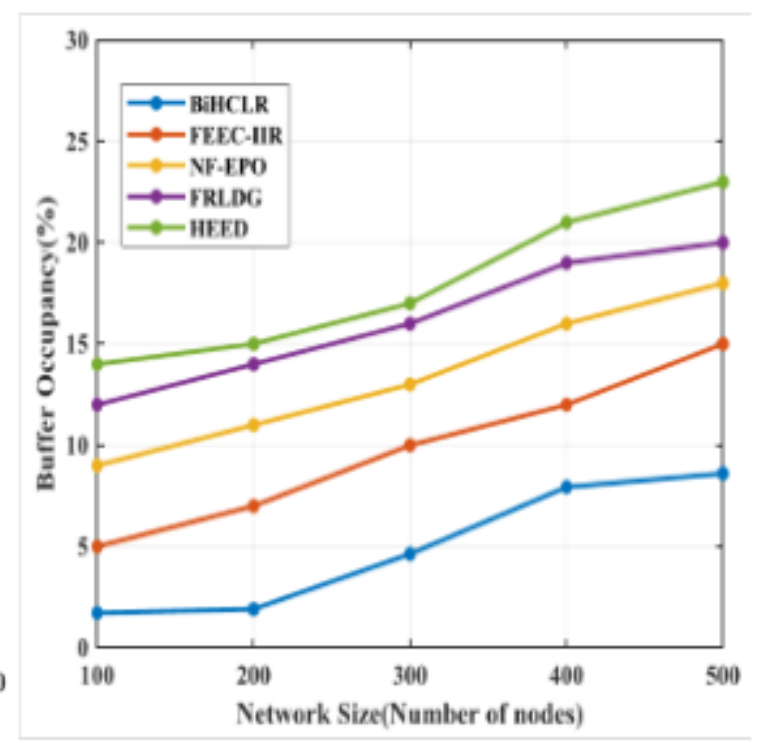

Fig. 15. Performance assessment of Buffer Occupancy

Fig. 15 shows the comparison of Buffer occupancy. Obtained buffer occupancy range in the proposed BiHCLR is low (2\%) in 100 nodes compared to other existing methods. The number of nodes increases the buffer occupancy range is also increases. In 100 nodes, the existing method approaches FEEC-IIR, NF-EPO, FRLDG, and HEED are 5\%, 9\%, 12\% and $14 \%$ respectively.

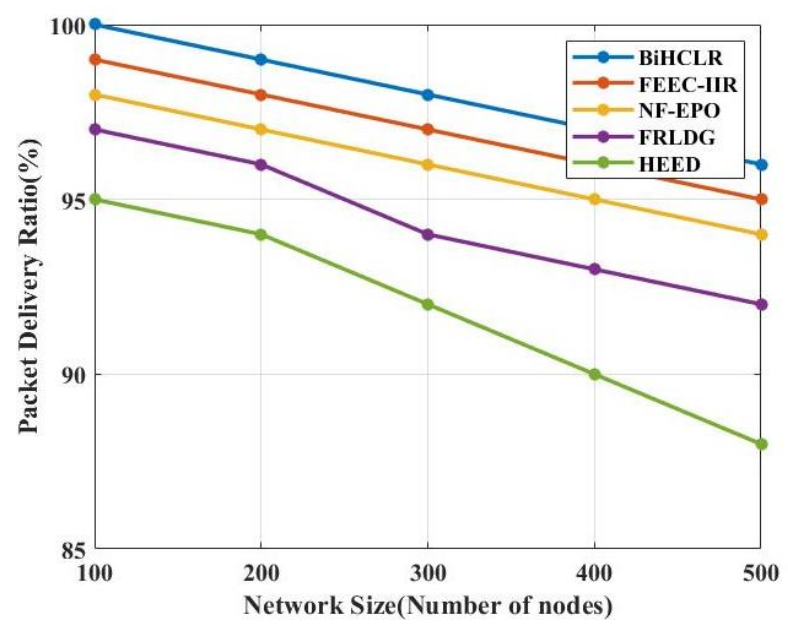

Fig. 16. Packet Delivery Ratio vs Network Size 
The total Packet Delivery Ratio (PDR) vs the Network Size is depicted in Fig. 16. As per the definition by IGI Global - PDR is defined as the ratio of the number of packets sent by the source node and the number of packets received by the destination node. The results show that despite the diminishing PDR when no. of nodes varies from 100 to 500, the proposed model outperforms other existing methods FEEC-IIR, NF-EPO, FRLDG, and HEED since within a limited sensor field, the density of the sensor nodes, the collision domain of each node widens and hence, the packet delivery weakens. From the above results, the proposed approach outperforms the compared results with respect to all the other parameters. Hence, this shows the significance of the proposed approach. The proposed BiHCLR approach has high PDR, throughput, and network lifetime. It has less delay, buffer occupancy, BER, packet loss, and energy consumption. These factors make the proposed approach a desirable algorithm to use in the current network for efficient routing [48-53].

\section{Conclusion}

This paper proposed a BiHCLR protocol for the effective routing in WSN assisted IoT. Initially, the deployed sensor nodes are arranged in grid forms, using the grid-based routing strategy. Then a fuzzy logic approach is used to select a $\mathrm{CH}$ in every cell of the grid. Then the hybrid moth search and salp swarm optimization is used to find the optimal path for the communication between $\mathrm{CH}$ and sink. The performance analysis shows that the proposed strategy consumed $30 \mathrm{~mJ}$ energy, with $0.99 \mathrm{Mbps}$ throughput, $2 \%$ BER, less than $2 \%$ packet loss, and buffer occupancy was $2 \%$. Moreover, the network resists up to 5600 rounds with an average transmission delay of $3.2 \mathrm{sec}$. Comparing the existing FEEC-IIR, NF-EPO, FRLDG, and HEED techniques the proposed BiHCLR protocol performed well. Ultimately suggested that the proposed BiHCLR technique with the combination of an intelligent algorithm and two bioinspired algorithms will be a better alternative for energy-based routing in WSN assisted IoT. However, the proposed technique does not concentrate on the direction of adjacent node selection, but it only concentrates on discovering the shortest path. So, it may lead to more iteration to determine the most suitable path. Hence in future, a better strategy instead of distance estimation should be proposed to enhance the execution speed of path discovery.

\section{References}

[1] D. Resner, G. Medeiros de Araujo, and A. A. Fröhlich, "Design and implementation of a crosslayer IoT protocol,” Science of Computer Programming, vol. 165, pp. 24-37, Nov. 2018. Article (CrossRef Link)

[2] W. Wei, H. Song, W. Li, P. Shen, and A. Vasilakos, "Gradient-driven parking navigation using a continuous information potential field based on wireless sensor network," Information Sciences, vol. 408, pp. 100-114, Oct. 2017. Article (CrossRef Link)

[3] H. Yetgin, K. T. K. Cheung, M. El-Hajjar, and L. Hanzo, “A Survey of Network Lifetime Maximization Techniques in Wireless Sensor Networks," IEEE Communications Surveys \& Tutorials, vol. 19, no. 2, pp. 828-854, 2017. Article (CrossRef Link)

[4] M. H. Anisi, G. Abdul-Salaam, M. Y. I. Idris, A. W. A. Wahab, and I. Ahmedy, "Energy harvesting and battery power based routing in wireless sensor networks," Wireless Networks, vol. 23, no. 1, pp. 249-266, Jan. 2017. Article (CrossRef Link) 
[5] S. Kurt, H. U. Yildiz, M. Yigit, B. Tavli, and V. C. Gungor, "Packet Size Optimization in Wireless Sensor Networks for Smart Grid Applications," IEEE Transactions on Industrial Electronics, vol. 64, no. 3, pp. 2392-2401, Mar. 2017. Article (CrossRef Link)

[6] R. H. Randhawa, A. Hameed, and A. N. Mian, "Energy efficient cross-layer approach for object security of CoAP for IoT devices," Ad Hoc Networks, vol. 92, p. 101761, Sep. 2019. Article (CrossRef Link)

[7] A. Hulbert, T. Kunicki, J. N. Hughes, A. D. Fox, and C. N. Eichelberger, "An experimental study of big spatial data systems," in Proc. of IEEE International Conference on Big Data (Big Data), pp. 2664-2671, 2016. Article (CrossRef Link)

[8] S. K. Gawali and M. K. Deshmukh, "Energy Autonomy in IoT Technologies," Energy Procedia, vol. 156, pp. 222-226, Jan. 2019. Article (CrossRef Link)

[9] Z. Fei, B. Li, S. Yang, C. Xing, H. Chen, and L. Hanzo, "A Survey of Multi-Objective Optimization in Wireless Sensor Networks: Metrics, Algorithms, and Open Problems," IEEE Communications Surveys and Tutorials, vol. 19, no. 1, pp. 550-586, 2017. Article (CrossRef Link)

[10] R. Bansal, S. Maheshwari, and P. Awwal, "Challenges and Issues in Implementation of Underwater Wireless Sensor Networks," Optical and Wireless Technologies, pp. 507-514, 2018. Article (CrossRef Link)

[11] A. Messaoudi, R. Elkamel, A. Helali, and R. Bouallegue, "Cross-layer based routing protocol for Wireless Sensor Networks using a fuzzy logic module," in Proc. of the $13^{\text {th }}$ International Wireless Communications and Mobile Computing Conference, pp. 764-769, June 2017. Article (CrossRef Link)

[12] S. Pudlewski, A. Prasanna, and T. Melodia, "Compressed-Sensing-Enabled Video Streaming for Wireless Multimedia Sensor Networks," IEEE Transactions on Mobile Computing, vol. 11, no. 6, pp. 1060-1072, June 2012. Article (CrossRef Link)

[13] C. Li, J. Wang, and M. Li, "Efficient Cross-Layer Optimization Algorithm for Data Transmission in Wireless Sensor Networks," Journal of Electrical and Computer Engineering, vol. 2015, pp. 16, 2015. Article (CrossRef Link)

[14] R. Singh and A. K. Verma, "Efficient image transfer over WSN using cross layer architecture," Optik (Stuttg), vol. 130, pp. 499-504, Feb. 2017. Article (CrossRef Link)

[15] M. Shelke, A. Malhotra, and P. N. Mahalle, "Congestion-Aware Opportunistic Routing Protocol in Wireless Sensor Networks," Smart Computing and Informatics, pp. 63-72, 2018. Article (CrossRef Link)

[16] J. Gubbi, R. Buyya, S. Marusic, and M. Palaniswami, "Internet of Things (IoT): A vision, architectural elements, and future directions," Future Generation Computer Systems, vol. 29, no. 7, pp. 1645-1660, Sep. 2013. Article (CrossRef Link)

[17] Y. Liu, K. Ota, K. Zhang, M. Ma, N. Xioung, and A. Liu, "QTSAC: An Energy-Efficient MAC Protocol for Delay Minimization in Wireless Sensor Networks,” IEEE Access, vol. 6, pp. 82738291, 2018. Article (CrossRef Link)

[18] V. Juneja and D. V. Gupta, "Security Against Vampire Attack in ADHOC Wireless Sensor Network: Detection and Prevention Techniques," in Proc. of International Conference on Wireless Intelligent and Distributed Environment for Communication, pp. 25-38, 2018. Article (CrossRef Link)

[19] Y. Zhou, Z. Sheng, C. Mahapatra, V. C. M. Leung, and P. Servati, "Topology design and crosslayer optimization for wireless body sensor networks," Ad Hoc Networks, vol. 59, pp. 48-62, May 2017. Article (CrossRef Link)

[20] J. Ben-Othman and B. Yahya, "Energy efficient and QoS based routing protocol for wireless sensor networks," Journal of Parallel Distributed Computing, vol. 70, no. 8, pp. 849-857, Aug. 2010. Article (CrossRef Link)

[21] M. Usman, N. Yang, M. A. Jan, X. He, M. Xu, and K. M. Lam, “A Joint Framework for QoS and QoE for Video Transmission over Wireless Multimedia Sensor Networks," IEEE Transactions on Mobile Computing, vol. 17, no. 4, pp. 746-759, Apr. 2018. Article (CrossRef Link)

[22] P. Park, P. Di Marco, and K. H. Johansson, "Cross-Layer Optimization for Industrial Control Applications Using Wireless Sensor and Actuator Mesh Networks," IEEE Transactions on 
Industrial Electronics, vol. 64, no. 4, pp. 3250-3259, Apr. 2017. Article (CrossRef Link)

[23] H. Pérez, J. J. Gutiérrez, S. Peiró, and A. Crespo, "Distributed architecture for developing mixedcriticality systems in multi-core platforms," Journal of Systems and Software, vol. 123, pp. 145159, Jan. 2017. Article (CrossRef Link)

[24] J. Wen, J. Yang, B. Jiang, H. Song, and H. Wang, "Big Data Driven Marine Environment Information Forecasting: A Time Series Prediction Network," IEEE Transactions on Fuzzy Systems, vol. 29, no. 1, pp. 4-18, Jan. 2021. Article (CrossRef Link)

[25] J. Yang, J. Zhang, and H. Wang, "Urban Traffic Control in Software Defined Internet of Things via a Multi-Agent Deep Reinforcement Learning Approach," IEEE Transactions on Intelligent Transportations Systems, pp. 1-13, 2020. Article (CrossRef Link)

[26] S. Pandey and D. Agrawal, "An ED-Based Enhanced Energy Efficient Cross Layer Model for Mobile Wireless Sensor Network,” National Academy Science Letters, vol. 40, no. 6, pp. 421-427, Dec. 2017. Article (CrossRef Link)

[27] A. S. Sadiq, T. Z. Almohammad, R. A. B. M. Khadri, A. A. Ahmed, and J. Lloret, "An EnergyEfficient Cross-Layer approach for cloud wireless green communications," in Proc. of the $2^{\text {nd }}$ International Conference on Fog and Mobile Edge Computing (FMEC), pp. 230-234, May 2017. Article (CrossRef Link)

[28] S. Munawwar, P. S. Kuntawar, and P. V. Roy, "Performance Analysis of Energy Efficient Cross Layer Load Balancing in Tactical Multi-gateway Wireless Sensor Network," International Research Journal of Engineering and Technology, vol. 4, no. 7, pp. 2834-2838, 2017. Article (CrossRef Link)

[29] S. K. S. L. Preeth, R. Dhanalakshmi, R. Kumar, and P. M. Shakeel, "An adaptive fuzzy rule based energy efficient clustering and immune-inspired routing protocol for WSN-assisted IoT system," Journal of Ambient Intelligence Humanized Computing, Dec. 2018. Article (CrossRef Link)

[30] S. K. S. L. Preeth, R. Dhanalakshmi, and P. M. Shakeel, "An intelligent approach for energy efficient trajectory design for mobile sink based IoT supported wireless sensor networks," Peerto-Peer Networking Applications, pp. 2011-2022, Aug. 2019. Article (CrossRef Link)

[31] A. Ben Ammar, A. Dziri, M. Terre, and H. Youssef, "Cross-Layer Approach Based Energy Minimization for Wireless Sensor Networks," Wireless Personal Communications, vol. 98, no. 2, pp. 2211-2221, Jan. 2018. Article (CrossRef Link)

[32] K. N. Dattatraya and K. R. Rao, "Hybrid based cluster head selection for maximizing network lifetime and energy efficiency in WSN," Journal of King Saud University-Computer and Information Sciences, Apr. 2019. Article (CrossRef Link)

[33] A. Amuthan and A. Arulmurugan, "Semi-Markov inspired hybrid trust prediction scheme for prolonging lifetime through reliable cluster head selection in WSNs," Journal of King Saud University-Computer and Information Sciences, July 2018. Article (CrossRef Link)

[34] S. A. Jesudurai and A. Senthilkumar, "An improved energy efficient cluster head selection protocol using the double cluster heads and data fusion methods for IoT applications," Cognitive Systems Research, vol. 57, pp. 101-106, Oct. 2019. Article (CrossRef Link)

[35] S. Janakiraman, "A Hybrid Ant Colony and Artificial Bee Colony Optimization Algorithm-based Cluster Head Selection for IoT," Procedia Computer Science, vol. 143, pp. 360-366, 2018. Article (CrossRef Link)

[36] T. M. Behera, S. K. Mohapatra, U. C. Samal, M. S. Khan, M. Daneshmand, and A. H. Gandomi, "Residual Energy-Based Cluster-Head Selection in WSNs for IoT Application," IEEE Internet of Things Journal, vol. 6, no. 3, pp. 5132-5139, June 2019. Article (CrossRef Link)

[37] D. Lin and Q. Wang, "An Energy-Efficient Clustering Algorithm Combined Game Theory and Dual-Cluster-Head Mechanism for WSNs,” IEEE Access, vol. 7, pp. 49894-49905, 2019. Article (CrossRef Link)

[38] H. El Alami and A. Najid, "ECH: An Enhanced Clustering Hierarchy Approach to Maximize Lifetime of Wireless Sensor Networks," IEEE Access, vol. 7, pp. 107142-107153, 2019. Article (CrossRef Link)

[39] Z. Lv, L. Qiao, J. Li, and H. Song, "Deep Learning Enabled Security Issues in the Internet of Things," IEEE Internet of Things Journal, 2020. Article (CrossRef Link) 
[40] Z. Lv, D. Chen, R. Lou, and H. Song, "Industrial Security Solution for Virtual Reality," IEEE Internet of Things Journal, 2020. Article (CrossRef Link)

[41] Z. Lv, "Virtual reality in the context of Internet of Things," Neural Computing and Applications, vol. 32, no. 13, pp. 9593-9602, July 2020. Article (CrossRef Link)

[42] Z. Lv and H. Song, "Mobile Internet of Things Under Data Physical Fusion Technology," IEEE Internet of Things Journal, vol. 7, no. 5, pp. 4616-4624, May 2020. Article (CrossRef Link)

[43] R. Logambigai, S. Ganapathy, and A. Kannan, "Energy-efficient grid-based routing algorithm using intelligent fuzzy rules for wireless sensor networks," Computers and Electrical Engineering, vol. 68, pp. 62-75, May 2018. Article (CrossRef Link)

[44] N. Mittal, "Moth Flame Optimization Based Energy Efficient Stable Clustered Routing Approach for Wireless Sensor Networks," Wireless Personal Communications, vol. 104, no. 2, pp. 677-694, Jan. 2019. Article (CrossRef Link)

[45] L. Hu, F. Hu, and S. Kumar, "Moth and Ant Inspired Routing in Hierarchical Airborne Networks with Multi-Beam Antennas," IEEE Transactions on Mobile Computing, vol. 18, no. 4, pp. 910922, Apr. 2019. Article (CrossRef Link)

[46] H. M. Kanoosh, E. H. Houssein, and M. M. Selim, "Salp Swarm Algorithm for Node Localization in Wireless Sensor Networks," Journal of Computer Networks Communications, vol. 2019, pp. 112, Feb. 2019. Article (CrossRef Link)

[47] G. G. Wang, "Moth search algorithm: a bio-inspired metaheuristic algorithm for global optimization problems," Memetic Computing, vol. 10, no. 2, pp. 151-164, June 2018.

Article (CrossRef Link)

[48] S. Bhushan, M. Kumar, P. Kumar, T. Stephan, A. Shankar, and P. Liu, "FAJIT: a fuzzy-based data aggregation technique for energy efficiency in wireless sensor network," Complex Intelligent Systems, 2021. Article (CrossRef Link)

[49] A. K. Singh, M. Alshehri, S. Bhushan, M. Kumar, O. Alfarraj, and K. R. Pardarshani, "Secure and energy-efficient data transmission model for WSN," Intelligent Automation \& Soft Computing, vol. 27, no. 3, 2021. Article (CrossRef Link)

[50] S. Bhushan, A. K. Singh, and S. Vij, "Comparative Study and Analysis of Wireless Mesh Networks on AODV and DSR," in Proc. of the $4^{\text {th }}$ International Conference on Internet of Things: Smart Innovation and Usages (IoT-SIU), pp. 1-6, 2009. Article (CrossRef Link)

[51] S. Bhushan, B. Bohara, P. Kumar, and V. Sharma, "A new approach towards IoT by using health care-IoT and food distribution IoT," in Proc. of the $2^{\text {nd }}$ International Conference on Advances in Computing, Communication, \& Automation, pp. 1-7, 2016. Article (CrossRef Link)

[52] P. Hemant, P. Kumar, and C. R. Nirmala, "Humor Identification from Short Texts Using Roberta and Albert," International Journal of Advanced Science and Technology, vol. 29, no. 04, pp. 95929600, 2020. Article (CrossRef Link)

[53] A. Jamshed, B. Mallick, and P. Kumar, "Deep learning-based sequential pattern mining for progressive database," Soft Computing, vol. 24, pp. 17233-17246, 2020. Article (CrossRef Link)

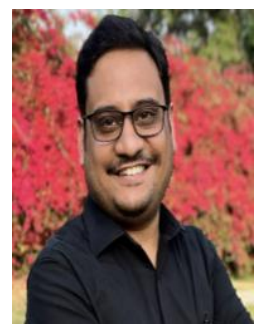

Aditya Tandon received his B.Tech. Degree in Computer Science Engineering from A.I.E.T. Jaipur (Rajasthan), India and M.Tech. Degree in Computer Science and Engineering from Amity University, Noida U.P., India. He has teaching experience of 6+ years. Presently, he is working as Assistant Professor in Department of CS\&E, Krishna Engineering College, Ghaziabad (affiliated by AKTU). He is also a Ph.D. research scholar at Quantum University, Roorkee. His research interests are in the field of Internet of Things, Artificial Intelligence, Wireless Sensor Networks and Network Security. So far, he has published 18 papers in reputed conferences and journals and 2 books - "Introduction to Artificial Intelligence using Python" and "Introduction to Machine Learning" so far. He is also running his YouTube channel "C 4 Yourself" having 18,000+ subscribers. 


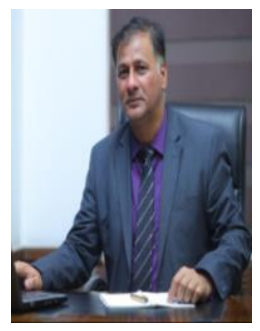

Professor (Dr.) Pramod Kumar has been working as Professor, Head (CSE) \& Dean (CSE \& IT) in Krishna Engineering College, Ghaziabad, since January 2018. He also served as Director of the Tula's Institute, Dehradun, Uttarakhand. He has more than 22 years of experience in academics. He completed his Ph.D. in Computer Science \& Engineering in 2011 and M.Tech. (CSE) in 2006. He is Senior Member of IEEE (SMIEEE) and Joint Secretary of IEEE U.P Section. He has published widely in international journals and conferences his research finding related to computer networks, Internet of Things(IoT), and machine learning. He has authored/co-authored more than 70 research papers and several chapters in edited books. He has supervised and co-supervised several M.Tech. and Ph.D. students. He has organized more than 10 IEEE international conferences and all the research papers of these conferences are now available in IEEE Xplore. He is the Editor of two books. He has conducted more than 15 Faculty Development Programs in the collaboration of EICT, IIT Roorkee, EICT, IIT Kanpur, and AKTU. He has organized IEEE National Workshop on research paper writing on March 27, 2017, and IEEE Women Symposium on February 21, 2016.

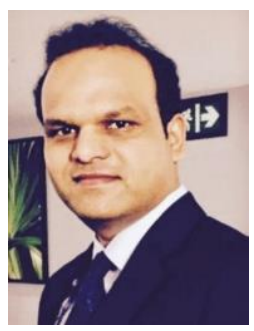

Prof. (Dr.) Vinay Rishiwal is working as Professor in Department of Computer Science and Information Technology, Faculty of Engineering and Technology, MJP Rohilkhand University, Bareilly. He has 20 years teaching and research experience. He received his Bachelor in Engineering (Computer Science and Engineering) in year 2000 from SRMS College of Engineering and Technology, Bareilly, India. He received his PhD (Computer Science and Engineering) in 2011 from Gautam Buddh Technical University, Lucknow, India. He has more than 80 research publications in peer reviewed journal and conferences. He has 12 patents into his account, out of which three are international patents and 09 are national patents. He is a reviewer/guest editor of many international journals. He has guided many students leading to M.E./M.Tech and Ph.D. His research interests include Quality of Service Issues in Advance Wireless Networks, Social networks, IoT, Machine Learning and Security aspects in cloud computing. He is a senior member of IEEE. He has worked as the convener of IEEE UP Section for Web/NL and Student Activities Committee. He is general/conference chair of many IEEE Conferences.

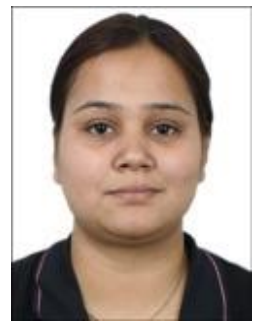

Mano Yadav is working as an Assistant Professor in the Department of Computer Science, Bareilly College, Bareilly, UP, India. She has 14 years teaching and research experience. She received her Master of Computer Application in 2007 from APEEJAY Institute of Technology, Greater Noida, India. She has received her Ph.D. in Computer Science and Information Technology in year 2012 from MJP Rohilkhand University, Bareilly, UP, India. She has more than 25 research publications in peer reviewed journals and conferences. She has five patents in her credit, out of which two international patents are granted and three national patents are published. Her research interest includes Wireless Sensor Networks, Machine Learning and IoT. She is a Technical member of many international conferences. She has served as the reviewer for many journals and a member of many technical societies like IACSIT-Singapore, MIR Labs USA etc.

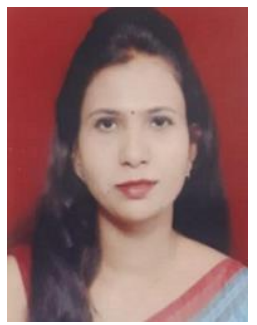

Preeti Yadav is working as Assistant Professor in Department of Computer Science and Information Technology, Faculty of Engineering and Technology, MJP Rohilkhand University, Bareilly. She has 13 years teaching and research experience. She received her Bachelor in Engineering (Computer Science and Engineering) in year 2007 from SRMS Collge of Engineering and Technology, Bareilly, India. She received her Masters in Engineering (Computer Science and Engineering) in year 2014 from Integral University, Lucknow, India She is pursuing her Ph.D. from IIT Roorkee, Uttarakhand, India. She has more than 15 research publications in journals and conferences. She has six patents in her credit, out of which four national patents are published and 2 international patents are granted. Her research interest includes Localization in Wireless Sensor Network, Machine Learning and IoT. She is a member of IEEE. 